\section{Feasibility of a novel web-based physical activity questionnaire for young children}

\author{
Stephanie E. Bonn,1 Pamela J. Surkan,2 \\ YIva Trolle Lagerros, ${ }^{3}$ Katarina Bälter ${ }^{1}$ \\ 1Department of Medical Epidemiology \\ and Biostatistics, Karolinska Institutet, \\ Sweden; 2Department of International \\ Health, Johns Hopkins Bloomberg School \\ of Public Health, USA, 3Department \\ of Medicine, Clinical Epidemiology Unit, \\ Karolinska Institutet, Sweden
}

\section{Abstract}

Accurate assessment of children's physical activity is critical in determining associations between childhood physical activity and health later in life. We have developed a web-based questionnaire, KidActive- $Q$, to assess physical activity in early childhood. The feasibility and reproducibility of KidActive- $Q$ were assessed in a sample of 20 children aged 2 to 6 years. The questionnaire was distributed to parents via e-mail on two occasions. The median time required to complete KidActive- $Q$ was 2.5 minutes. All the parents reported that the questionnaire was easy or very easy to fill. The intraclass correlations for test-retest reliability were 0.60 (95\% confidence interval: $0.31-0.88$ ) for daily time spent outdoors, and 0.85 (95\% confidence interval: $0.72-0.97)$ for daily time watching television. Our results suggest that KidActive- $Q$ is a user-friendly tool for assessing physical activity in early childhood. To our knowledge, this is the first web-based questionnaire specifically developed for young children.

\section{Introduction}

Sedentary behavior and low levels of physical activity in youth has been associated with higher levels of body fat, ${ }^{1}$ and physical activity patterns initiated in childhood have been shown to persist throughout life. ${ }^{2}$ To better understand the role of physical activity during childhood, and its association to body composition and overweight, this complex behavior must be assessed accurately. However, this assessment is complicated by children's highly variable activity patterns, i.e. physical activity is often in short bursts, the velocity and type of movement vary considerably, and they have frequent rest periods. ${ }^{3}$
Paper-based questionnaires have previously been used in most large studies, but increased accessibility to the Internet has made webbased data collection possible.4,5 Since young children themselves cannot respond to a questionnaire and report duration, frequency or intensity of activities performed, proxy reports from parents or another caregiver are used. ${ }^{6} \mathrm{~A}$ limited number of questionnaires for assessing physical activity in children are available, ${ }^{7-10}$ and none have, to the best of our knowledge, been developed specifically for the Web. To fill the need of a web-based questionnaire assessing usual physical activity in early childhood, we have developed KidActive-Q. The main aim of the present study is to describe KidActive- $Q$, and to report its feasibility and user-friendliness. Further, the reproducibility of KidActive$\mathrm{Q}$ was assessed by comparing two administrations of the web-based questionnaire.

\section{Materials and Methods}

\section{Study participants}

In May 2009, all children ( $\mathrm{n}=28$, aged 2 to 6 years, $54 \%$ girls) attending a daycare group in a suburb of Stockholm, Sweden, and their parents were invited, and agreed, to participate in the present study. Parents were given both written and oral information about the study and provided written informed consent prior to enrollment. The weight $(\mathrm{kg})$ and height $(\mathrm{cm})$ of the children were measured at the beginning of the study. The new web-based physical activity questionnaire for children, KidActiveQ, was administered to the parents on two occasions separated by about three weeks. The study was approved by the Research Ethics Committee at Karolinska Institutet, Stockholm, Sweden.

\section{KidActive- $Q$ questionnaire}

KidActive- $Q$ is a web-based proxy questionnaire assessing usual physical activity patterns among young children. A parent or other adult closely associated with the child is asked to report the child's usual physical activity during the past few months. KidActive- $Q$ is an interactive questionnaire, and 10 to 12 questions are answered, depending on the response pattern. The questions assess the overall level of physical activity, method of transportation to and from daycare, time spent outdoors, time spent watching television and/or playing computer games, and athletic activities performed on a regular basis. All questions have predefined answers regarding different activity level, frequency and/or duration. Responses to the questionnaire can thereafter be summarized into a score reflecting the activity level of the child. An example of the questionnaire layout is shown in Figure 1.
Correspondence: Stephanie E. Bonn, Department of Medical Epidemiology and Biostatistics, Karolinska Institutet, Nobels väg 12 A, 17177 Stockholm, Sweden.

Tel. +46.852482298 - Fax: +46.8314975

E-mail: stephanie.bonn@ki.se

Key words: children, epidemiology, internet, physical exercise.

Acknowledgements: the authors would like to thank Tanja Siiteri and Kristina Lundqvist for their important contributions to data collection. We also thank the daycare personnel, and the children and their parents for their participation in the study.

Funding: this work was supported by grants to LifeGene from AFA insurance, Torsten and Ragnar Söderbergs Foundation, Karolinska Institutet, Stockholm's County Council, and the Swedish Research Counsil.

Contributions: SEB, YTL, KB, contributed substantially to the design and acquisition of data; SEB, drafted the article; PJS, YTL, KB, critically revised it for important intellectual content.

Conflict of interests: the authors report no conflict of interests.

Received for publication: 5 June 2012.

Revision received: 27 September 2012.

Accepted for publication: 28 September 2012

This work is licensed under a Creative Commons Attribution NonCommercial 3.0 License (CC BYNC 3.0).

(C) Copyright S.E. Bonn et al., 2012

Licensee PAGEPress, Italy

Pediatric Reports 2012; 4:e37

doi:10.4081/pr.2012.e37

\section{Study design}

The first KidActive- $Q$ was sent out via e-mail on day one of the study and the second was administered three weeks later. Individual user names and passwords served as identifiers in the web-questionnaire program that automatically recorded the response time to the questionnaire. The reproducibility of KidActive- $Q$ was assessed by comparing the results obtained on the two occasions. Immediately following completion of the questionnaire on the first occasion, the parents were asked to respond to questions regarding the user-friendliness of KidActive- $Q$. Parents were asked to assess whether they thought the questionnaire was easy to fill out, and if they thought the questions were relevant and presented in a suitable sequence. Lastly, the parents were asked to rate the questionnaire on a scale from 1 (worst) to 5 (best). 


\section{Statistical methods}

Paired t-tests were used to assess potential differences in characteristics between boys and girls. The reproducibility of questions assessing overall activity, time spent outdoors, and time spent watching television, was assessed using intraclass correlation coefficients. ${ }^{11}$ The significance level was set to $\mathrm{P}<0.05$. Analyses were performed using STATA 11.1 (STATA Corporation, College Station, TX, USA).

\section{Results}

Of the 28 children and parents who agreed to participate in the study, 20 completed the KidActive- $Q$ questionnaire on both occasions, and were included in the assessment of feasibility and reproducibility. Characteristics of the children are presented in Table 1 . Descriptive results from the first KidActive- $Q$ questionnaire are given in Table 2. The median years of birth of the mothers and fathers were 1971 and 1968, respectively. All parents were highly educated, with 39 out of 40 having a university education. No differences in characteristics were found between boys and girls.

The intraclass correlation for test-retest reliability of KidActive- $Q$ for the question on overall physical activity level was 0.66 [95\% confidence interval (CI): 0.41-0.91], while the intraclass correlation for total time spent outdoors per day was 0.60 (95\% CI: 0.31-0.88), and for total time spent watching television per day 0.85 (95\% CI: 0.72-0.97).

The mean time required for the parents to fill out the KidActive- $Q$ questionnaire on the first occasion was 2 minutes and 37 seconds, while corresponding time on the second occasion was 1 minute and 50 seconds. All responding parents reported that the questionnaire was easy or very easy to answer and agreed or fully agreed that the order of the questions presented was good. Eighteen out of 20 responding parents agreed or fully agreed that the questions were relevant. The remaining two reported that they had no opinion regarding the relevancy. The mean overall score, on a scale from 1 (worst) to 5 (best), was 4.4 $( \pm 0.7)$, and 18 out of the 20 of the parents gave a score of 4 or 5 .

\section{Discussion}

Our results indicate that KidActive- $Q$ is a user-friendly and reproducible instrument, which is feasible for web-based data collection on the physical activity of young children.

Only a limited number of questionnaires intended to assess different dimensions of
Table 1. Characteristics of children included in the study, $n=20(50 \%$ girls $)$.

\begin{tabular}{lcccc} 
& Mean & Standard deviation & Min & Max \\
Age, years & 4.15 & 1.3 & 2 & 6 \\
Weight, $\mathrm{kg}$ & 18.2 & 4.2 & 10.9 & 28.8 \\
\hline Height, $\mathrm{cm}$ & 106.0 & 11.8 & 82.0 & 127.5 \\
Body mass index, $\mathrm{kg} / \mathrm{m}^{2}$ & 17.6 & 3.2 & 12.0 & 25.5 \\
\hline
\end{tabular}

Table 2. Descriptive data based on responses to some questions in the KidActive-Q on the first occasion $(n=20)$.

\begin{tabular}{|c|c|c|c|c|}
\hline Activity & Time per day & Minutes & Number & $\%$ \\
\hline Watching television & $\begin{array}{l}\text { Weekdays } \\
\text { Weekends }\end{array}$ & $\begin{array}{c}0 \\
30 \\
90 \\
180 \\
0 \\
30 \\
90 \\
180\end{array}$ & $\begin{array}{c}1 \\
12 \\
7 \\
0 \\
2 \\
2 \\
14 \\
2\end{array}$ & $\begin{array}{c}5 \\
60 \\
35 \\
0 \\
10 \\
10 \\
70 \\
10\end{array}$ \\
\hline Using the computer & $\begin{array}{l}\text { Weekdays } \\
\text { Weekends }\end{array}$ & $\begin{array}{c}0 \\
30 \\
0 \\
30\end{array}$ & $\begin{array}{c}18 \\
2 \\
17 \\
3\end{array}$ & $\begin{array}{l}90 \\
10 \\
85 \\
15\end{array}$ \\
\hline Playing outdoor & $\begin{array}{l}\text { Weekdays } \\
\text { Weekends }\end{array}$ & $\begin{array}{c}90 \\
180 \\
300 \\
360 \\
90 \\
180 \\
300 \\
360\end{array}$ & $\begin{array}{c}6 \\
11 \\
3 \\
0 \\
2 \\
10 \\
6 \\
2\end{array}$ & $\begin{array}{c}30 \\
55 \\
15 \\
0 \\
10 \\
50 \\
30 \\
10\end{array}$ \\
\hline
\end{tabular}

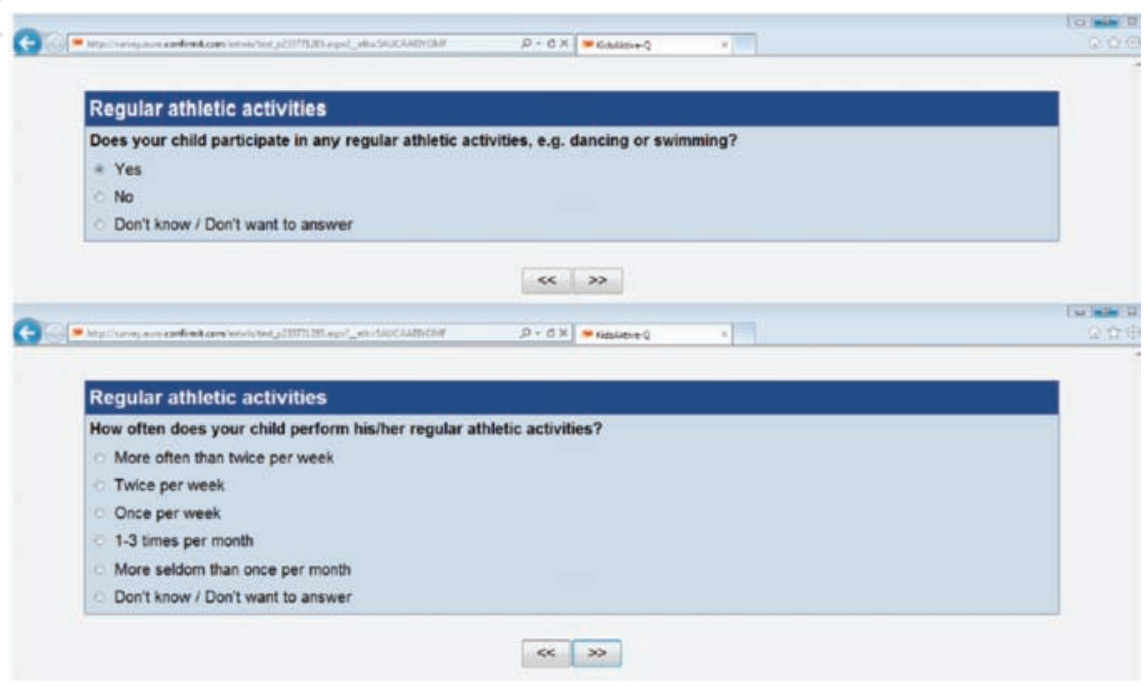

Figure 1. Screenshots of KidActive-Q showing a question regarding the performance of regular athletic activities, and a follow-up question if the response to the initial question is Yes. 
physical activity in children have been validated. Corder et al. ${ }^{8}$ found a significant correlation $(r=0.42)$ between minutes per week of moderate to vigorous physical activity using the Children's Physical Activity Questionnaire and accelerometer measurements in a sample of 27 children with a mean age of 4.9 years. The relative validity of the Netherlands Physical Activity Questionnaire was assessed by Jantz et al. ${ }^{9}$ in sample of 204 children with a mean age of 5.7 years. Comparing an arbitrary numerical score of the questionnaire against measurements from accelerometers, significant correlations were found for total $(r=0.33)$ and vigorous activity $(r=0.36)$. Burdette et al. ${ }^{7}$ have reported a significant association $(r=0.20)$ between parental reports of preschool children's outdoor playtime and a direct measure of physical activity using accelerometers in a sample of 250 preschool children with a mean age of 3.7 years.

In the present study, data were collected in a small and selected sample of children, with highly educated parents. This created a homogenous group limiting the generalizability of the results due to the fact that the sample was not representative of the whole population. Results from this study should therefore be interpreted with caution. All children attended daycare, as do most young children in Sweden, ${ }^{12}$ so this characteristic is not a limitation in a Swedish population. The wide confidence intervals for the intraclass correlation point estimates that indicate test-retest repeatability, may partly be explained by the small sample size and sampling variability. Nonetheless, the point estimates do indicate good reproducibility. The reproducibility of specific questions in KidActive- $Q$ was assessed by comparing the results of the questionnaire administered on two occasions approximately three weeks apart. This time interval was chosen as it would be long enough to ensure that the parents would not remember their answers from the previous questionnaire administration, and short enough to ensure that the children's physical activity had not changed between the two occasions.

KidActive- $Q$ assesses the activity patterns of children and gives an overall picture of whether a child is physically active or inactive. Marked developmental advances occur through the early childhood years, affecting the kind of activities children are able to perform, and posing a challenge to any instrument evaluating the physical activity of children in this age group. Because of differences in activity patterns during this period, the results from KidActive- $Q$ must be interpreted while bearing in mind the age of the child. However, stratification by age was outside the scope of our analysis due to the small sample size. To ensure the validity of KidActive- $Q$, results from the questionnaire should be compared with those from other objective and thoroughly validated measures, e.g. the doubly labeled water method or acceleromters, depending on the dimension of physical activity to be validated. Larger validation studies are needed to assess both the criterion and construct validity of KidActive-Q.

\section{Conclusions}

In conclusion, our results suggest that KidActive- $Q$ is a modern and easily applied tool for assessing physical activity among young children. The questionnaire was developed for and is currently being used in an ongoing large prospective cohort study. 13 To the best of our knowledge, this is the first report on a questionnaire for assessing physical activity among young children specifically developed for webbased use.

\section{References}

1. Marshall SJ, Biddle SJ, Gorely T, et al. Relationships between media use, body fatness and physical activity in children and youth: a meta-analysis. Int $\mathrm{J}$ Obes Relat Metab Disord 2004;28:1238-46.

2. Malina RM. Physical activity and fitness: pathways from childhood to adulthood. Am
J Hum Biol 2001;13:162-72.

3. Oliver M, Schofield GM, Kolt GS. Physical activity in preschoolers: understanding prevalence and measurement issues. Sports Med 2007;37:1045-70.

4. Balter KA, Balter O, Fondell E, Lagerros YT. Web-based and mailed questionnaires: a comparison of response rates and compliance. Epidemiology 2005;16:577-9.

5. Ekman A, Litton JE. New times, new needs; e-epidemiology. Eur J Epidemiol 2007;22:285-92.

6. Welk GJ, Corbin CB, Dale D. Measurement issues in the assessment of physical activity in children. Res Q Exerc Sport. 2000; 71:59-73.

7. Burdette HL, Whitaker RC, Daniels SR. Parental report of outdoor playtime as a measure of physical activity in preschoolaged children. Arch Pediatr Adolesc Med 2004;158:353-7.

8. Corder K, van Sluijs EM, Wright A, et al. Is it possible to assess free-living physical activity and energy expenditure in young people by self-report? Am J Clin Nutr 2009; 89:862-70.

9. Janz KF, Broffitt B, Levy SM. Validation evidence for the Netherlands physical activity questionnaire for young children: the Iowa bone development study. Res $Q$ Exerc Sport 2005;76:363-9.

10. Telford A, Salmon J, Jolley D, Crawford D. Reliability and validity of physical activity questionnaires for children: the children's leisure activities study survey (CLASS). Pediatr Exerc Sci 2004;16:64-78.

11. Lee J, Koh D, Ong CN. Statistical evaluation of agreement between two methods for measuring a quantitative variable. Comput Biol Med 1989;19:61-70.

12. Dunér B, Westerholm A. Barns Omsorg 2005. Report no. 307. Stockholm: The Swedish National Agency for Education; 2007.

13. Almqvist C, Adami HO, Franks PW, et al. LifeGene - a large prospective populationbased study of global relevance. Eur $\mathrm{J}$ Epidemiol 2011;26:67-77. 